\title{
Morphological and Electromyogram Analysis for the Spinal Accessory Nerve Transfer to the Suprascapular Nerve in Rats
}

\author{
Jun Yan $^{1}$, Kazuhito Ogino ${ }^{1,2}$, Jiro Hitomi ${ }^{1}$ \\ ${ }^{1}$ Department of Anatomy, School of Medicine, Iwate Medical University, \\ Iwate, Japan \\ ${ }^{2}$ Department of Plastic Surgery, Dokkyo Medical University, \\ Tochigi, Japan \\ E-mail: junyan@iwate-med.ac.jp \\ Received April 19, 2011; revised June 3, 2011; accepted June 25, 2011
}

\begin{abstract}
For many years, nerve transfer has been commonly used as a treatment option following peripheral nerve injury, although the precise mechanism underlying successful nerve transfer is not yet clear. We developed an animal model to investigate the mechanism underlying nerve transfer between branches of the spinal accessory nerve (Ac) and suprascapular nerve (Ss) in rats, so that we could observe changes in the number of motor neurons, investigate the 3-dimensional localization of neurons in the anterior horn of the spinal cord, and perform an electromyogram (EMG) of the supraspinatus muscle before and after nerve transfer treatment. The present experiment showed a clear reduction in the number of $\gamma$ motor neurons. The distributional portion of motor neurons following nerve transfer was mainly within the neuron column innervating the trapezius. Some neurons innervating the supraspinatus muscle also survived post-transfer. Compared with the non-operated group, the EMG restoration rate of the supraspinatus muscle following nerve transfer was $60 \%$ in the experimental group and $80 \%$ in a surgical control group. Following nerve transfer, there was a distinct reduction in the number of $\gamma$ motor neurons. Therefore, $\gamma$ motor neurons may have important effects on the recovery of muscular strength following nerve transfer. Moreover, because the neurons located in regions innervating either the trapezius or supraspinatus muscle were labeled after Ac transfer to Ss, we also suggest that indistinct axon regeneration mechanisms exist in the spinal cord following peripheral nerve transfer.
\end{abstract}

Keywords: Nerve Transfer Treatment, Fluorescent Dye Labeling, Electromyogram, Nerve Axonal Regeneration, Rat

\section{Introduction}

Traditionally, direct suture treatment was considered necessary for the treatment of peripheral nerve injury $[1,2]$. However, peripheral nerve regeneration was thought to be impossible because successful cases were very rare prior to the 18th century. According to Green's operative hand surgery (third edition), the earliest successful cases were demonstrated at the end of the 19th century [3]. The first case of peripheral nerve transfer was reported by Balance, and indicated that voluntary movement of mimetic muscles could be recovered by suturing the spinal accessory nerve (Ac) or hypoglossal nerve to the facial nerve [2]. This cure for peripheral nerve injury was called nerve transfer, and was commonly used thereafter for facial nerve paralysis or many other types of peripheral nerve injury [4-9].

Nerve transfer as a cure for paralysis of the brachial plexus was also a classic treatment, and good treatment results were evident [10-13]. In a commonly used clinical treatment, the branch innervating the trapezius (Ac) was transferred to the suprascapular nerve (Ss) to recover paralysis of the supraspinatus muscle and, finally, to recover function of the humeral joint. However, the morphological mechanism underlying this method was not clarified. A phenomenon was observed under electron microscopy in which nerve growth cones from the nodes of Ranvier on damaged nerve fibers could enter into the 
peripheral side of the nerve for re-innervation [14-16]. Following this, many researchers attempted to clarify the mechanisms underlying nerve fiber regeneration. However, changes in the motor neurons of the anterior horn, which are known to play a key role during regeneration or re-innervation of damaged peripheral nerve fibers, remain unaddressed.

In the 1970s, a retrograde method using horseradish peroxidase (HRP) was the main method used to investigate motor neurons in the anterior horn [17-27]. However, a major flaw was that HRP leaked, raising concern over the reliability of such assessments [28].

Fluorescent dye (DiI) proved to be a highly reliable neuronal marker and for use in pathway tracing [29]. The fluorescent dye dissolves in the lipids of Schwann cells; therefore, the reliability of the dye is better than HRP, which could diffuse into the surrounding tissue and be absorbed directly into the axon [29,30]. Using the fluorescent dye (DiI), we performed experiments in adult rats and proved its reliability as a retrograde tracer in the peripheral nerve system. [31-35].

The objectives of the present experiments were to 1) use an animal model to confirm the relationship between nerve transfer between the spinal accessory nerve (Ac) and suprascapular nerve (Ss) with changes in the number and distribution of motor neurons; 2) construct a 3-dimensional image of motor neurons (including Ac and Ss) in the anterior horn and compare it to changes in neuron localization; and 3) to observe changes in the electromyogram (EMG) of the supraspinatus muscle before and after nerve transfer treatment.

\section{Materials and Methods}

Adult Wistar rats ( $\hat{\jmath}, 9$ weeks of age; 105 - $250 \mathrm{~g}$ ) were used in all experiments. All animals were obtained from Japan SLC Inc., and were maintained in the Animal Care Service Center, School of Medicine, Iwate Medical University. Animals were handled in compliance with ethical guidelines. Animals were anesthetized with an initial intraperitoneal dose of sodium pentobarbital $(40 \mathrm{mg} / \mathrm{kg}$ ) and maintained with additional $2.5 \mathrm{mg}$ doses as needed.

\subsection{Nerve Transfer Operations (Ac to $S s, N=4$, Age 9 Weeks)}

As shown in Figures 1 and 2, the muscular branch innervating the branch innervating the trapezius (Ac) and suprascapular (Ss) nerves were dissected and removed under surgical microscopy (Olympus Optical Co) in 4 rats. The proximal section of the Ac was then transferred to the distal section of the Ss using the epineurium sewing method. The animals were maintained for 9 weeks then killed and their spines removed [36].

\subsection{Control Group (Ac to Ac, $N=4$ )}

A comparison of the regeneration results following transfer of the Ac to the Ss with that of sewing the same nerve (Ac to Ac) was considered a necessary control for our study. In order to create appropriate control experiments, the Ac was dissected and amputated in 4 rats then the proximal and distal sections were sewed together using the same nerve transfer method.

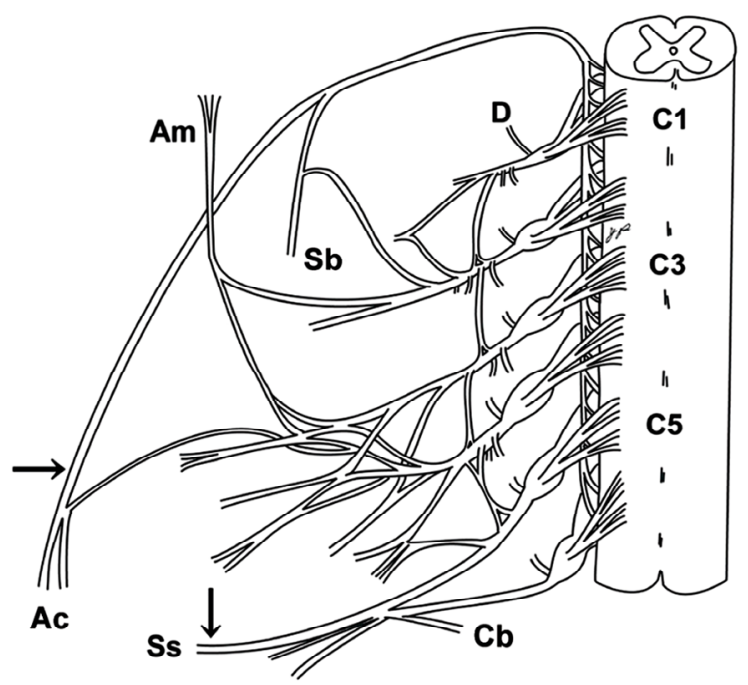

(a)

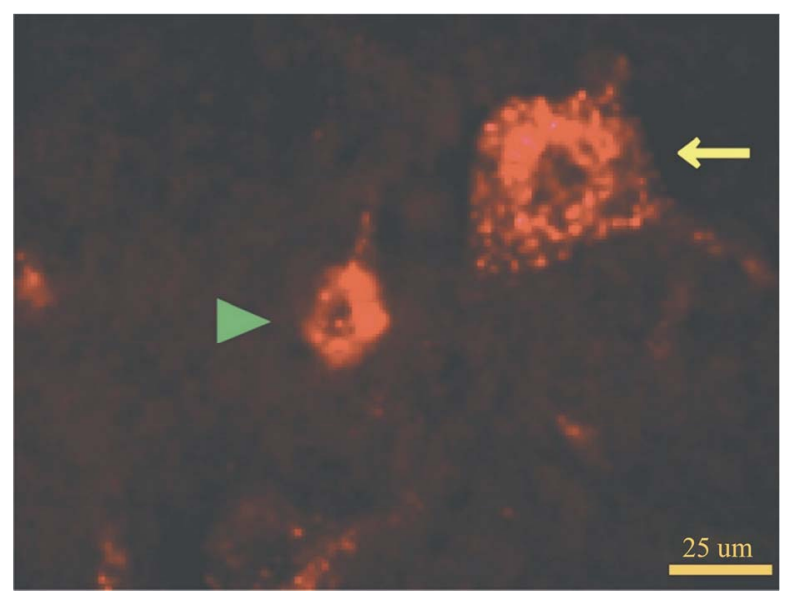

(b)

Figure 1. The form of Ac and cervical plexus (sketch) and $\alpha$, $\gamma$ motor neuron in anterior horn (photo). (a): The peripheral part of the Ac and cervical nerves of the rat (ventral view). The Ac has two main branches to innervate the sternocleidomastoideus and trapezius. $\mathrm{C2}, \mathrm{C} 3$, and $\mathrm{C} 4$ send a branch directly to the Ac mainly. Black arrows: the dyed regions of the Ac and Ss; (b): The photo showing the $\alpha$ motor neuron (yellow arrow) and $\gamma$ motor neuron (green arrowhead) in anterior horn. 


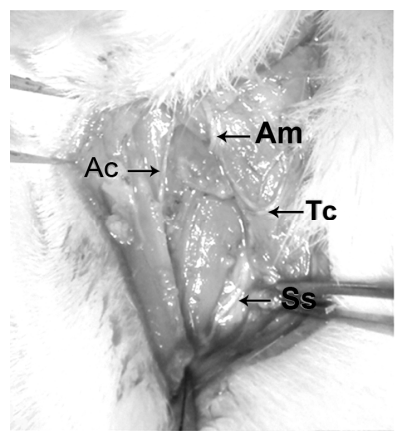

(a)

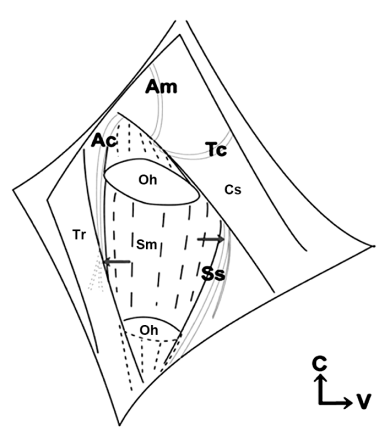

(c)

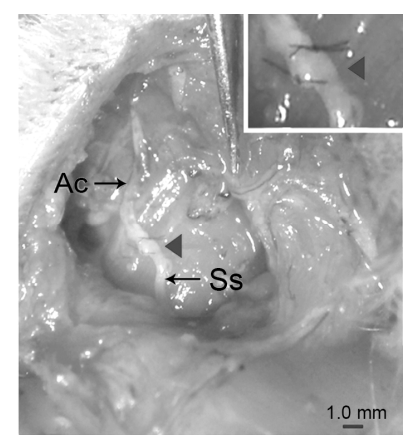

(b)

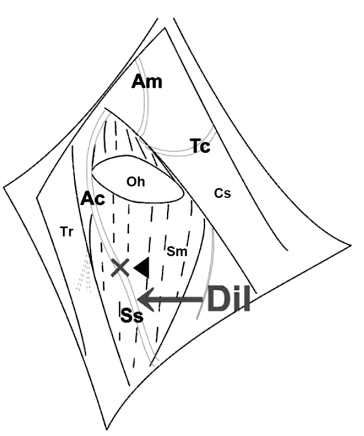

(d)
Figure 2. The photos and sketches showing the operation of the dye treatment and nerve transfer (Ac to Ss). (a): the Ac and $\mathrm{Ss}$ are in the upper and inferior edge of the omohyoideus muscle in rat. (b): the omohyoideus was amputated, the Ac and Ss has been transferred (black arrowhead). The right upper is the extended photo of the transferred nerve (Ac to Ss). (c): the red arrows are showing the dyed regions of the Ac and Ss, respectively. (d): the red-cross indicates the transferred point of two nerves, and the red arrow is showing the dyed point after nerve transfer (after operation 9 weeks).

\subsection{Electromyogram (Ss, $N=4$; Ac to Ac, $N=4$; Ac to $S s, N=4$; All Animals were 18 Weeks of Age)}

In order to evaluate the regeneration of the nerve fibers in the transferred nerves, EMGs of the supraspinatus muscle was examined in the non-operated, nerve transfer, and control groups. The nerve innervating the muscle was dissected and the EMG recorded using the electrode stimulation method (Chart for Windows 5.5.1, ADInstruments, Lexington, Australia). In all cases, the stimulation points were changed on the surface of the muscle and the total number of stimulations was 30 . The highest average value was then calculated.

\subsection{Observation of Motor Neurons in the \\ Anterior Horn of the Ac and $\mathrm{Ss}$ (Ac, $\mathrm{N}=4$; Ss, $N=4$; Ac to Ac, $N=4$; Ac to $S s, n=4$ )}

Under surgical microscopy, the Ac, Ss (non-operated group, 18 weeks of age), and the nerve transfer group ( 9 weeks after surgery) were dissected and amputated at a point near the muscle or on the distal portion of the operated point, respectively. The fluorescent dye, DiI [1,1'dioctadecyl-3,3,3',3'-tetramethylind carbocyanine perchlorate; diI-C18-(3)] was applied to the proximal section of the nerves. Proximal sections were wrapped with the surrounding connective tissue, and the distal sections were burned to prevent ambiguous labeling. Two weeks after surgery, animals were anesthetized with $50 \mathrm{mg} / \mathrm{kg}$ intraperitoneal sodium pentobarbital and fixed by intracardiac perfusion with $150 \mathrm{ml}$ physiological salt solution and $300 \mathrm{ml} 4 \%$ paraformaldehyde in $0.1 \mathrm{M}$ phosphate buffer. The spinal cord (C1 to Th1 segments) was surgically removed along with its dorsal root ganglia. Specimens were post-fixed in the same fixing solution for $24 \mathrm{~h}$ then placed serially into $10 \%, 20 \%$, and $30 \%$ sucrose solutions in $0.1 \mathrm{M}$ phosphate buffer for $24 \mathrm{~h}$, respectively. Each spinal cord segment was formed into one block and cut transversely into $50-\mu \mathrm{m}$ serial sections using a microtome. All sections were observed and photographed using a laser confocal microscope (LSM200GBSU2; Olympus Optical Co. Ltd., Japan).

We used 4 animals to confirm the restoration of the peripheral nerves. To do this, peripheral nerve specimens (distal part of the transferred nerve point) were removed. Specimens were then embedded in paraffin, cut transversely, and stained using the Masson-Trichrome method [37].

Morphological assessment of the samples was a valuable aspect of our investigation (Figure 1). The diameter of all stained motor neurons was measured to judge the proportion of $\alpha$ and $\gamma$ motor neurons in each segment (over $30-\mu \mathrm{m}$ was defined as $\alpha$ and under $25 \mu \mathrm{m}$ was defined as $\gamma$ motor neurons [14]).

The number of $\alpha$ and $\gamma$ motor neurons in each segment were counted, and the average value of each segment (in 4 spinal cord specimens) was calculated for each. Then the average number of $\alpha$ and $\gamma$ motor neurons in the Ac (non-operated group) and Ac transferred to Ss groups were treated with the t-test for medical statistics.

In three animals (one from each of the nerve transfer, control, and un-operated groups), all images were reconstructed with 3-dimensional reconstruction software (VoxBlast 3.1, Vaytec, Fairfield, USA) to compare the localized distribution of the motor neurons in the anterior horn of the spinal cord.

\section{Results}

\subsection{Electromyogram (EMG)}

An EMG (supraspinatus muscle) analysis was conducted 
in the three groups. In the non-operated group (Ss), the average maximum value of the EMG was $0.045 \mathrm{mv}$, And was $0.037 \mathrm{mv}$ in the control group (Ac to Ac), and 0.030 $\mathrm{mv}$ in the nerve transfer group (Ac to Ss) (Table 3). The EMG restoration rate in the control group (Ac to Ac) was $80 \%$ of the non-operated group, whilst the restoration rate in the nerve transfer group (Ac to Ss) was $60 \%$ of the non-operational group.

\subsection{Microscopic Observation of Peripheral Nerve Regeneration Following Nerve Transfer}

As shown in Figure 3, a definite reduction in the number of nerve fibers on histological sections was not observed in nerve transfer or non-operational group. Permeation of inflammatory cells in the nerve transfer group was not observed. However, the inside diameter of the myelinated nerve fibers was reduced, and atrophy of the myelin sheath was apparent. Moreover, we observed the disappearance of axon(s) in a small number of sections.

\subsection{Localization of Neurons in the Anterior Horn and 3-Dimensional Reconstruction of the Neurons}

The motor neurons of the Ac were distributed from the $\mathrm{C} 2$ to $\mathrm{C} 7$ segments, and formed a longitudinal column. In sections of cranial segments, the neurons were located in the lateral portion, but in caudal segments the neurons seemed to be located in the medial portion of the anterior horn. However, 3-dimensional reconstruction showed that the motor neurons formed a straight column from the cranial to caudal segments (Figure 4(a)). The neurons of the Ss were distributed predominantly from the C3 to C7 segments, and were located in the dorsal-lateral portion of the anterior horn, forming a longitudinal column (Figure 4(b)). Results showed that the distribution of motor neurons in the Ac and Ss slightly overlapped in $\mathrm{C} 3$ and $\mathrm{C} 4$, but in the caudal segments the distribution of the two columns was separate from each other (Figures 4(a), 4(b), and 4(d)).

On the other hand, after nerve transfer the distribution of motor neurons were broadly consistent with that in the Ac. We speculate that the motor neurons within the transferred nerve originated from those belonging to the Ac. In caudal segments, however, some motor neurons were located in the dorsal-lateral portion (belonging to Ss), although with reduced frequency (Figure 4(d)).

\subsection{Number and Classification of Labeled Motor Neurons}

In the non-operated rats (including Ac and Ss), the loca- tion and number of $\alpha$ and $\gamma$ motor neurons were confirmed after retrograde labeling with the fluorescent DiI. The number of $\alpha$ and $\gamma$ motor neurons within the Ac, Ss, control group (Ac to Ac), and the transfer group (Ac to Ss) are shown in Table 1. The number of $\gamma$ motor neurons in the nerve transfer group were definitely reduced, although $\alpha$ neurons were also reduced. The ratio of $\alpha: \gamma$ was $4.8: 1$ in Ss, and $7.3: 1$ in the nerve transfer group (Table 1). In particular, the number of the $\gamma$ motor neurons in caudal segments were significantly reduced (C5, C6, and C7) compared with cranial segments.

As shown in Table 2, after nerve transfer the restoration rates of $\alpha$ and $\gamma$ motor neurons were $79 \%$ and $64 \%$, respectively. In the control group, the restoration rates of the two neuron groups were $85 \%$ and $84 \%$, respectively.

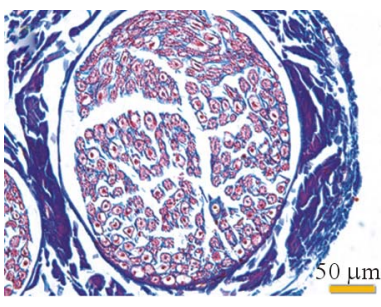

(a)

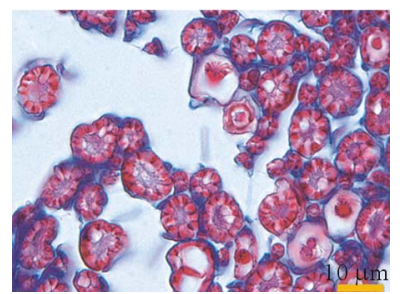

(c)

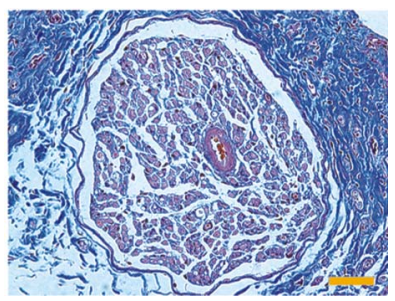

(e)

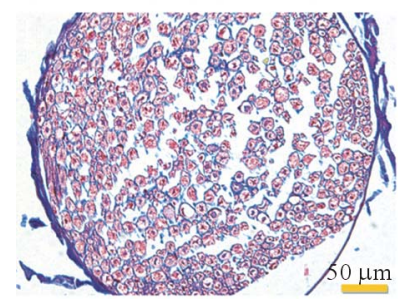

(b)

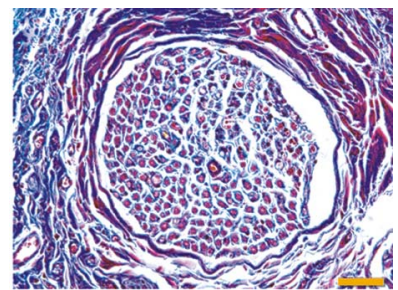

(d)

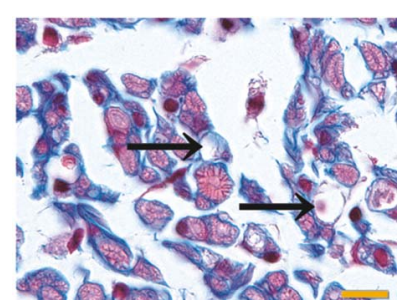

(f)
Figure 3. The section photos of peripheral nerves (un-perational and after nerve transfer). (a) (Ac) and (b) (Ss) are showing the peripheral nerve sections of un-operational cases. (d) and (e) are showing the sections which from the cranial and caudal part of the transferred nerve, respectively. It could be confirmed that the diameter of myelinated fibers was reduced, after compared with (a) and (b). (c) and (f) are the extended photos of (b) and (e). Note some of demyelinisation (black arrows) were observed and the number of fibers were decrease, the inflammatory cells were observed in (f). 


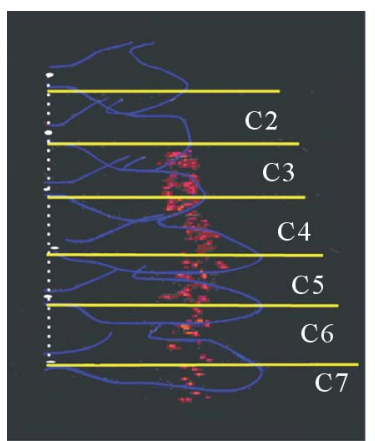

(a)

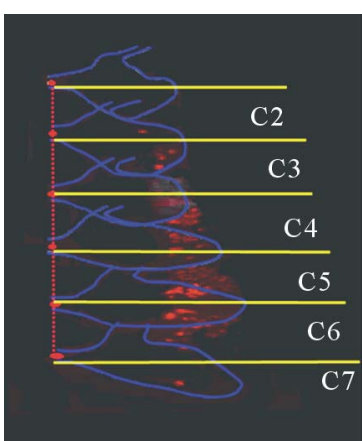

(c)

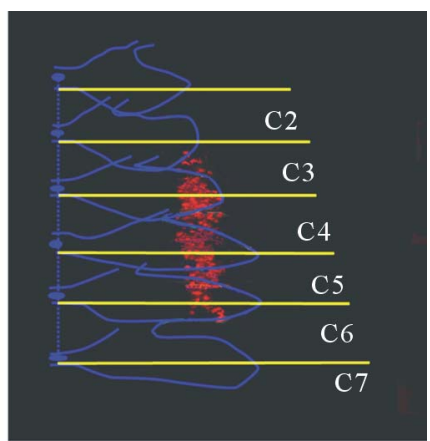

(b)

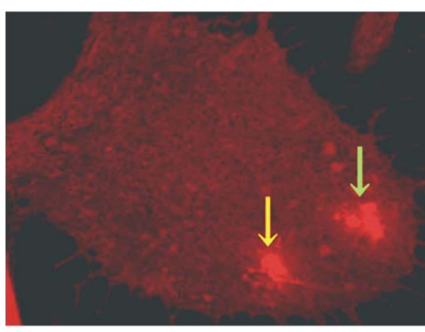

(d)
Figure. 4. The neurons distribution of Ac, Ss and Ac to Ss. The neurons of Ac were located in the ventral portion of anterior horn and that of Ss were in the dorsal-lateral portion (yellow arrow: Ac; green arrow: Ss; in (d). After 3-D reconstruction, the neuron column of Ac was showing in $A$, that of Ss was in $B$ and the column after nerve transfer was showing in (c). It was clear that the column after nerve transfer is similar with the Ac, although some dyed neurons were also observed (d).

Table 1. The average number of the $\alpha$ and $\gamma$ motor neurons. The average number of the $\alpha$ and $\gamma$ motor neurons in Ac (un-operation), Ss (un-operation), Ac-Ac (control) and AcSs (nerve transfer) were showing in the table. After nerve transfer, the numbers of the $\alpha$ and $\gamma$ motor neurons were decreased clearly. After analysis by t-test, the signification difference between the two values were approved (in both $\alpha$ : Ac-Ac/Ac and $\gamma$ : Ac-Ss/Ac, $p<0.01$ ).

\begin{tabular}{cccc}
\hline & $\alpha$ & $\gamma$ & $\alpha / \gamma$ \\
\hline Ac & 233.0 & 39.0 & $5.9 / 1$ \\
Ss & 222.0 & 45.0 & $4.8 / 1$ \\
Ac-Ac & 199.0 & 33.0 & $6.0 / 1$ \\
Ac-Ss & 183.0 & 25.0 & $7.3 / 1$ \\
\hline
\end{tabular}

\section{Discussion}

Peripheral nerve transfer has been used for surgical treatment of brachial plexus injury for many years $[2,11,13,38]$. In particular, the "standard" method of
Table 2. The restoration rate of the $\alpha$ and $\gamma$ motor neurons. After nerve transfer, the restoration rate of the $\alpha$ and $\gamma$ motor neuron were $79 \%$ and $64 \%$, respectively. In the control, the rates of the two neuron group were $85 \%$ and $84 \%$, respectively.

\begin{tabular}{ccc}
\hline & $\alpha$ & $\gamma$ \\
\hline Ac-Ss/ Ac*100\% & $79 \%$ & $64 \%$ \\
Ac-Ac/ Ac*100\% & $85 \%$ & $84 \%$ \\
\hline
\end{tabular}

Table 3. The EMG of supraspinatus muscle in nerve transfer cases, control cases and un-operational cases. The graph is showing the un-operational (a), control (b) and transfer cases (c) of No.3 animal. The restoration rate of EMG in control cases (Ac to $\mathrm{Ac}$ ) is $\mathbf{8 0 \%}$ of the un-operational cases, and the rate of EMG in the nerve transfer (Ac to $\mathrm{Ss}$ ) is $60 \%$ of the un-operational cases.

\begin{tabular}{cccc}
\hline & $\begin{array}{c}\text { Un-operation } \\
(\mathrm{Ss})\end{array}$ & $\begin{array}{c}\text { control } \\
(\mathrm{Ac}-\mathrm{Ac})\end{array}$ & $\begin{array}{c}\text { transfer } \\
\text { Ac-Ss }\end{array}$ \\
\hline 1 & 0.045 & 0.036 & 0.031 \\
2 & 0.044 & 0.038 & 0.029 \\
3 & 0.047 & 0.035 & 0.031 \\
4 & 0.044 & 0.037 & 0.029 \\
Aver. & 0.045 & 0.037 & 0.030 \\
\hline
\end{tabular}

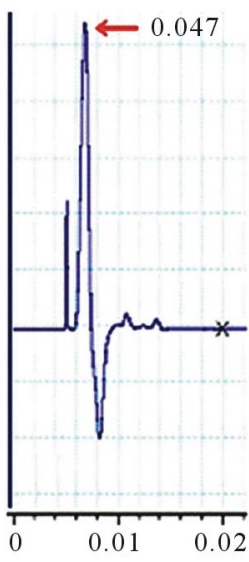

(a)

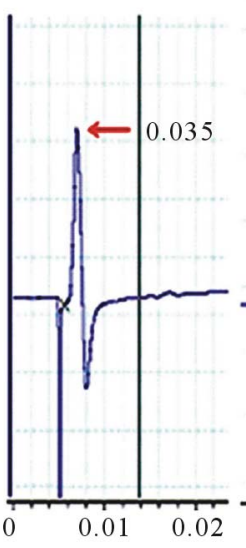

(b)

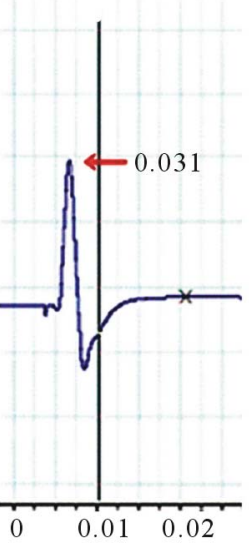

(c) medical treatment for upper root injury of the brachial plexus employs the transfer of the muscular branch innervating the trapezius to the suprascapular nerve $[5,9,39-41]$. In recent years, the quality of surgical microscopy has improved, not only for nerve transfer but also in terms of the suturing method used for peripheral nerve injury [42]. However, there is a clear difference in the results for individual patients, and it is difficult to judge treatment results clinically after nerve transfer. On the other hand, observing the motor neurons in the anterior horn has proven to be effective in animal models for 
estimating peripheral nerve reconstruction [14,21,37]. However, these methods are not sufficient to fully explain the outcome of the nerve transfer operation. In the present experiment, we developed an animal model to evaluate the outcome of peripheral nerve transfer operations. In the present experiment, we developed an animal model to order to evaluate the outcome of peripheral nerve transfer operations. With this animal model, we investigated the specific number and distribution of motor neurons in the Ac and Ss groups following nerve transfer in each cervical segment. We also generated 3-dimensional reconstructions to compare the distribution of the motor neurons before and after nerve transfer treatment.

Our results show that the neuron column of Ac begins from $\mathrm{C} 2$ to $\mathrm{C} 7$ (mostly in $\mathrm{C} 3$ to $\mathrm{C} 6$ ), but that of Ss originates from $\mathrm{C} 4$ to $\mathrm{C} 7$ (mostly in $\mathrm{C} 5$ and $\mathrm{C6}$ ). The distribution of motor neurons innervating different muscles could be used to judge the origin of the muscles [43] and we believe that the transfer of nerves innervating the same original muscles could lead to improved treatment outcomes. In the present experiment, the two columns were separated and, according to the literature, the two muscles could originate from different muscle masses [43]. Consequently, this may explain why the contraction strength of the supraspinatus muscle was only restored by $60 \%$.

We investigated the regeneration outcome of nerve transfer 9 weeks after surgery. This interval ( 9 weeks) was considered to represent a suitable term for observing nerve regeneration because an amputated peripheral nerve can extend axons by $4.3 \mathrm{~mm} /$ day [44]. In this experiment, regenerated fibers were mostly represented by myelinated fibers. We did not observe the presence of inflammatory cells. Consequently, nerve regeneration occurred in a satisfactory manner, although reportedly $90 \%$ of neurons in humans can degenerate in just six months following surgery $[28,45,46]$.

In the present experiments, the restoration rate of the $\alpha$ and $\gamma$ motor neurons were $85 \%, 84 \%$ (Ac to Ac) and $79 \%, 64 \%$ (Ac to Ss), and in reference to the EMG we believe our results are exact. We did not find any reports showing a $90 \%$ restoration of contraction of the supraspinatus muscle after nerve transfer treatment. Therefore, we believe that our animal model is an important and successful model. Misdirection of $\alpha$ motor neurons in the anterior horn after nerve transfer has been observed with the HRP method [18,37]. However, in the present experiment it is not yet clear why some motor neurons innervating the supraspinatus muscle survived (in this model, the axons of the Ss were amputated, and the foundation for regeneration was lost). The present experiment focused on changes in motor neuron numbers and the distributional proportions of $\alpha$ and $\gamma$ motor neurons before and after peripheral nerve transfer. Our results clearly show that the number of $\gamma$ motor neurons was lower following nerve transfer than before nerve transfer. These observations agree with previous studies of $\alpha$ motor neurons; the $\gamma$ motor neurons have not previously been included in analyses $[18,37]$. The number of $\gamma$ motor neurons was significantly reduced following nerve transfer. Therefore, the $\gamma$ motor neurons could be presumed to have a very important effect on the functional restoration of damaged muscles.

We believe the reduction in $\gamma$ motor neurons is the main factor underlying restoration of muscle contraction. If the number of $\gamma$ motor neurons is reduced, then the contraction conditions of the intra-fusal fibers in the spindles could not be transmitted to $\alpha$ motor neurons in a smooth manner. More specifically, decreased efficiency of the $\gamma$ loop would result in reduced contractile strength. In considering why the number of $\gamma$ motor neurons was reduced after nerve transfer, we speculate that several factors are involved. Firstly, the axons of the $\gamma$ motor neurons are thin and non-myelinated. Secondly, the specific effects of neutrophic factors are not yet proven on the neurons [47]. Supplementary observation of associated changes of sensory neurons in spinal ganglia is necessary.

The motor neurons innervating the trapezius and supraspinatus muscle were observed together in C3-C6, and the two groups of neurons were identified in a somewhat ventral-dorsal arrangement. Following nerve transfer, the surviving motor neurons were observed predominantly in the same distributional field of Ac, and this phenomenon could not be explained by the theory of "motor neuron misdirection" [37]. On the other hand, the effects of homeobox genes are very important when axons extend out of the spinal cord in early embryos [48-51]. However, these early molecular studies did not consider whether the neurons could survive (or not) following nerve transfer. Therefore, it is necessary to clarify why the motor neurons innervating the supraspinatus muscle survived after nerve transfer treatment; i.e., by adopting a genetic or molecular approach.

\section{Conclusions}

The distributional portion of motor neurons following nerve transfer was mainly within the neuron column innervating the trapezius. Some neurons innervating the supraspinatus muscle also survived post-transfer. The EMG restoration rate of the supraspinatus muscle following nerve transfer was $60 \%$, and the rate of the control group was $80 \%$ of that in the non-operated group. Following nerve transfer, there was a distinct reduction 
in the number of $\gamma$ motor neurons. Therefore, $\gamma$ motor neurons may have important effects on the recovery of muscular strength following nerve transfer treatment.

After nerve transfer treatment, the functional restoration of damaged muscle may be related to the developmental origin of the donor nerve.

\section{Acknowledgments}

We thank Dr. S. Kobayashi, and Dr. S. Kimura (Iwate Medical University) for their technical advice.

This work was supported by a research grant from the Ministry of Education, Culture, and Science of Japan (No. 22590178), and it was also supported financially by the Advanced Medical Science Center of Iwate Medical University.

\section{References}

[1] J. Aboujaoude, J. Y. Alnot and C. Oberlin, "The Spinal Accessory Nerve (N. Accessories) 1: Anatomical Study," Revue de Chirurgie Orthopedique et Reparatrice de l Appareil Moteur, Vol. 80, No. 3, 1994, pp. 291-296.

[2] S. C. Ballance, and A. B. Duel, "The Operative Treatment Official Palsy: By the Introduction of Nerve Grafts into the Fallopian Canal and by other Intratemporal Methods," Archives of Otolaryngology, Vol. 15, No. 1, 1932, pp. 1-70.

[3] E. F. Wilgis and M. T. Brushart, "Nerve Repair and Grafting," Green's operative hand surgery, 3rd Edition, Churchill Livingstone, New York, 1993, pp. 1321-1322.

[4] J. A. Bertelli, and M. F. Ghizoni, "Reconstruction of C5 and C6 Brachial Plexus Avulsion Injury by Multiple Nerve Transfers: Spinal Accessory to Suprascapular, Ulnar Fascicles to Biceps Branch, and Triceps Long or Lateral Head Branch to Axillary Nerve," Journal of Hand Surgery-American Volume, Vol. 29, No. 1, 2004, pp. 131139. doi:10.1016/j.jhsa.2003.10.013

[5] A. B. Jayme and F. G. Marcos, "Reconstruction of C5 and C6 Brachial Plexus Avulsion Injury by Multiple Nerve Transfer: Spinal Accessory to Suprascapular, Ulnar Fascicles to Biceps Branch, and Triceps Long or Lateral Head Branch to Axillary Nerve," Journal of Hand Surgery-American Volume, Vol. 29, No. 1, 2004, pp. 131139. doi:10.1016/j.jhsa.2003.10.013

[6] M. J. Malessy, G. C. de Ruiter, K. S. de Boer and R. T. Thomeer, "Evaluation of Suprascapular Nerve Neurotization after Nerve Graft or Transfer in the Treatment of Brachial Plexus Traction Lesions," Journal of Neurosurgery, Vol. 101, No. 3, 2004, pp. 377-389. doi:10.3171/jns.2004.101.3.0377

[7] Y. Nakatsuchi, N. Ishigaki, N. Ogiwara, Y. Tateiwa and T. Matsunaga, "Treatment for Brachial Plexus Palsy with Upper Root Injuries," Journal of Japanese Society for Surgery of the Hand, Vol. 21, No. 2, 2004, pp. 193-196.

[8] C. Oberlin, D. Béal, S. Leechavengvongs, A. Salon, M. C.
Dauge and J. J. Sarcy, "Nerve Transfer to Biceps Muscle Using a Part of Ulnar Nerve for C5-C6 Avulsion of the Brachial Plexus: Anatomical Study and Report of Four Cases," American Journal of Surgery, Vol. 19, No. 2, 1994, pp. 232-237. doi:10.1016/0363-5023(94)90011-6

[9] Y. Taniwaki, M. Noguchi, H. Matsusaki and T. Tani, "Flexor Plasties of the Elbow after Brachial Plexus Injury," Journal of Japanese Society for Surgery of the Hand, Vol. 22, No. 3, 2005, pp. 597-601.

[10] Y. Hattori, K. Doi, S. Toh and A. S. Baliarsing, "Surgical Approach to the Spinal Accessory Nerve for Brachial Plexus Reconstruction," Journal of Hand SurgeryAmerican Volume, Vol. 26, No. 6, 2001, pp. 1073-1076.

[11] A. Lurje, "Concerning Surgical Treatment of Traumatic Injury of the Upper Division of the Brachial Plexus (Erb's-type)," Annals of Surgery, Vol. 127, No. 2, 1948, pp. 317-326. doi:10.1097/00000658-194802000-00009

[12] H. Nakajima, K. Uchida, T. Inukai, S. Kobayashi and H. Baba, "Retrograde Transfection of Neurotrophic Factor Gene after Cervical Spinal Cord Injury," Central Japan Journal of Orthopaedic Surgery and Traumatology, Vol. 52, No. 2, 2009, pp. 161-162.

[13] H. J. Seddon, "Nerve Grafting," Journal of Bone and Joint Surgery (British Volume), Vol. 45, No. 3, 1963, pp. 447-461.

[14] T. M. Brushart and M. M. Mesulam, "Altenation in Connections between Muscle and Anterior Horn Motoneurons after Peripheral Nerve Repair," Science, Vol. 208, No. 4444, 1980, pp. 603-605. doi:10.1126/science. 7367884

[15] C. IDe, "Peripheral Nerve Regeneration," Neuroscience Research, Vol. 25, No. 1, 1996, pp. 101-121.

[16] C. IDe and S. Kato, "Peripheral Nerve Regeneration," Neuroscience Research (Suppl), Vol. 13, 1990, pp. S157S164.

[17] R. E. Burke, P. L. Strick, K. Kanda, C. C. Kim and B. Walmsley, "Anatomy of Medial Gasutrocnemius and Soleus Motor Nuclei in Cat Spinal Cord," Journal of Neurophysiology, Vol. 40, No. 3, 1977, pp. 667-680.

[18] N. Ito, H. Konishi, T. Nakamura, T. Tsukazaki, K. Maeda and K. Iwazaki, "Experimental Study of Direct Nerve Implantation in Denervated Muscle - Histological Study of Spinal Anterior Horn Cells by Horseradish Peroxidase Method," Journal of Japanese Society for Surgery of the Hand, Vol. 7, No. 1, 1991, pp. 70-73.

[19] S. Kitamura and A. Sakai, "A Study on the Localization of Sternocleidomastoid and Trapezius Motoneurons in the Rat by Means of the HRP Method," Anatomical Record, Vol. 202, No. 4, 1982, pp. 527-536. doi:10.1002/ar.1092020412

[20] H. Konishi, "The Experimental Study of the Assessment of the Peripheral Nerve Function by the Horseradish Peroxidase Method," Journal of the Japanese Orthopaedic Association, Vol. 63, No. 5, 1989, pp. 810-818.

[21] S. Nicolopoulos-Stournaras and F. J. Iles, "Motor Neuron Columns in the Lumber Spinal Cord of the Rat," Journal of Comparative Neurology, Vol. 217, No. 1, 1983, pp. 
75-85. doi:10.1002/cne. 902170107

[22] S. Ohta, "Erythropoietin Prevents Motor Neuron Death after Rat Spinal Root Avulsion," Journal of Japanese Society for Surgery of the Hand, Vol. 24, No. 5, 2008, pp. 1008-1011.

[23] J. M. Peyronnard and L. Charron, "Decreased Horseradish Peroxidase Labeling in Deafferented Spinal Motoneurons of the Rat," Brain research, Vol. 275, No. 2, 1983, 203-214. doi:10.1016/0006-8993(83)90983-6

[24] M. Saito, "Spatial Distribution of Motoneurons Innervating Elbow Flexor and Extensor Muscles of Upper-Arm in the Rat Spinal Cord," Journal of the Japanese Orthopaedic Association, Vol. 60, No. 5, 1986, pp. 1167-1174.

[25] K. Tada, S. Ohshita, K. Yonenobu, K. Ono and N. Shimizu, "Development of Spinal Motoneuron Innervations of the Upper Limb Muscle in the Rat," Experimental Brain Research, Vol. 35, No. 2, 1979, pp. 287-293. doi:10.1007/BF00236616

[26] K. Xu, K. Uchida, H. Nakajima, S. Kobayashi and H. Baba, "Targeted Retrograde Transfection of Adenovirus Vector Carrying Brain-Derived Neurotrophic Factor Gene Prevents Loss of Mouse (twy/twy) Anterior Horn Neurons in Vitro Sustaining Mechanical Compression," Spine, Vol. 31, No. 17, 2006, pp. 1867-1874. doi:10.1097/01.brs.0000228772.53598.cc

[27] J. Ygge, "On the Organization of the Thoracic Spinal Ganglion and Nerve in the Rat," Experimental Brain Research, Vol. 55, No. 3, 1984, pp. 395-401. doi:10.1007/BF00235269

[28] C. L. Smith, "The Developmentand Postnatal Organization of Motor Nuclei in the Rat Thoracic Spinal Cord," Journal of Comparative Neurology, Vol. 220, No. 1, 1983, pp. 16-28. doi:10.1002/cne.902200104

[29] M. G. Honig and R. I. Hume, "DiI and DiO: Versatile Fluorescent Dyes for Neuronal Labeling and Pathway Tracing," Trends in Neurosciences, Vol. 12, No. 2, 1989, pp. 333-314. doi:10.1016/0166-2236(89)90040-4

[30] A. Hayashi, A. Yanai and Y. Komuro, "End-to-Side Neurorrhaphy with Nerve Grafting: Analysis Using Fluorescent Dye (DiI) as a Neural Tracer," Journal of Japan Society of Plastic and Reconstructive Surgery, Vol. 23, No. 1, 2003, pp. 6-14. (English Abstract)

[31] J. Yan, R. W. Tian and M. Horiguchi, "Distribution of Sensory Neurons of Ventral and Dorsal Cervical Cutaneous Nerves in Dorsal Root Ganglia of Adult Rat: A Double-Label Study Using DiO and DiI," Okajimas Folia Anatomica Japonica, Vol. 79, No. 5, 2002, pp. 129-134. doi:10.2535/ofaj.79.129

[32] J. Yan and M. Horiguchi, "Fiber Arrangements of Cutaneous and Muscular Rami in Cervical Nerves of the Rat: Regarding Conception of the Spinal Nerve Stratum-Structure," Okajimas Folia Anatomica Japonica, Vol. 79, No. 4, 2002, pp. 107-112. doi:10.2535/ofaj.79.107

[33] J. Yan and J. Hitomi, "Analysis of Motor Fibers in the Communicating Branch Between the Cervical Nerves and the Spinal Accessory Nerve to Innervate Trapezius in the Rat," Okajimas Folia Anatomica Japonica, Vol. 83, No. 3, 2006, pp. 77-84. doi:10.2535/ofaj.83.77
[34] J. Yan, Y. Aizawa and J. Hitomi, "Localization of Motoneurons that Extend Axons through the Ventral Rami of Cervical Nerves to Innervate the Trapezius Muscle: A Study Using Fluorescent Dyes and 3D Reconstruction Method," Clinical Anatomy, Vol. 20, No. 1, 2007, pp. 41 -47. doi:10.1002/ca.20300

[35] J. Yan and J. Hitomi, "Fiber Arrangement of Nerves Belonging to Ventral and Dorsal Divisions in the Proximal Region of the Brachial Plexus: A Study Using Fluorescence of DiI and DiO in Adult Rats," Surgical and Radiologic Anatomy, Vol. 26, No. 4, 2004, pp. 312-318. doi:10.1007/s00276-003-0222-y

[36] E. Wada, Y. Akaboshi, K. Ikeda and Y. Sakaguchi, "Experimental Study of the Motor Neuron Misdirection after Peripheral Nerve Transfer: HRP Method," Journal of Orthopaedic Surgery and Traumatology, Vol. 12, No. 2, 1985, pp. 309-312. (Japanese)

[37] Y. Sano, "Histological Technics: Theoretical and Applied," Nanzando Company limited, Tokyu, 2003, pp. 200-201. doi:10.1097/00006534-200207000-00018

[38] W. D. Xu, Y. D. Gu, J. G. Xu and L. J. Tan, "Full-Length Phrenic Nerve Transfer by Means of Video-Assisted Thoracic Surgery in Treating Brachial Plexus Avulsion Injury," Plastic and Reconstructive Surgery, Vol. 110, No. 1, 2002, pp. 104-109. doi:10.1097/00006534-200207000-00018

[39] S. B. Guan, C. 1. Hou, D. S. Chen and Y. D. Gu, "Restorration of Shoulder Abduction by Transfer of the Spinal Accessory Nerve to Suprascapular Nerve Through Dorsal Approach: A Clinical Study," Chinese Medical Journal, Vol. 119, No. 9, 2006, pp. 707-712.

[40] J. K. Terzis and I. Kostas, "Suprascapular Nerve ReconStruction in 118 Cases of Adult Posttraumatic Brachial Plexus," Plastic and Reconstructive Surgery, Vol. 117, No. 2, 2006, pp. 613-629. doi:10.1097/01.prs.0000203410.35395.fa

[41] S. F. Wang, G. M. Zhang, Y. D. Gu, L. Y. Zhang and X. Zhao, "A Clinical Study of Spinal Accessory Nerve Transfer for the Repair of Suprascapular Nerve to Restore the Impaired Abduction Function of the Shoulder Following Avulsion Injury of Brachial Plexus," Chinese Journal of Orthopaedics, Vol. 20, No. 3, 2000, pp. 594597.

[42] Z. H. Dailiana, H. Mehdian and A. Gilbert, "Surgical Anatomy of spinal Accessory Nerve: Is Trapezius Functional Deficit Inevitable after Division of the Nerve?" Journal of Hand Surgery, Vol. 26, No. 2, 2001, pp. 137141.

[43] C. U. A. Kappers, G. C. Huber and E. C. Crosby, "The Comparative Anatomy of the Nervous System of Vertebrates, Including Man,” Macmillan, New York, 1936.

[44] Y. Komiya, "Axonal Regeneration and Axonal Transport," Orthopaedic \& Traumatic Surgery, Vol. 25, No. 8, 1982, pp. 1353-1363.

[45] C. G. Zhang, J. J. Ma, G. Terenghi, C. Mantovani and M. Wiberg, "Phrenic Nerve Transfer in the Treatment of Brachial Plexus Avulsion: An Experimental Study of Nerve Regeneration and Muscle Morphorogy in Rats," 
Microsurgery, Vol. 24, No. 3, 2004, pp. 232-240. doi:10.1002/micr.20015

[46] C. G. Zhang, G. Terenghi, C. Mantovani and M. Wiberg, "Neuronal Survival, Regeneration and Musclemorphology after Posterior C7 nerve Transfer: An Experimental Study," Plastic and Reconstructive Surgery, sVol. 59, No. 2, 2006, pp. 717-725.

[47] R. Shibata-Iwasaki, H. Dekimoto, Y. Katsuyama, S. Kikkawa and T. Terashima, "Anterograde Labeling of the Corticospinal Tract in jimpy Mutant Mice by DiI Injection into the Motor Cotex," Archives of Histology and Cytology, Vol. 70, No. 5, 2007, pp. 297-301. doi:10.1679/aohc.70.297

[48] J. Briscoe, L. Sussel, P. Serup, D. Hartigan- O'Connor, T. M. Jessell, J. L. Rubenstein and J. Ericson, "Homeobox

\section{List of Abbreviations}

Ac: the branch innervating trapezius;

Am: auricularis magnus;

C: cranial;

$\mathrm{Cb}$ : communicating branch to $\mathrm{C} 7$;

$\mathrm{C} 1$ : the first cervical segment.

Cs: cleidomastoideus (in rat);

D: dorsal branch;
Gene NKx2.2 and Specification of Neuronal Identify by Graded Sonic Hedgehog Signaling," Nature, Vol. 398, No. 6728, 1999, pp. 622-627.doi:10.1038/19315

[49] K. Kristensson and Y. Olsson, "Retrograde Axonal Transport of Protein," Brain research, Vol. 29, No. 2, 1971, pp. 363-365. doi:10.1016/0006-8993(71)90044-8

[50] O. Pabst, J. Rummelies, B. Winter and H. H. Arnold, "Targeted Disruption of the Homeobox Gene Nkx2.9 Reveals a Role in Development of the Spinal Accessory nerve," Development, Vol. 130, No. 6, 2003, pp. 11931202. doi: $10.1242 /$ dev.00346

[51] O. Uemura and H. Okamoto, "The Islet-1 Family in Subtype Specification of Motor Neurons," Advances in Neurological Sciences, Vol. 49, No. 1, 2005, pp. 25-34.

Oh: omohyoideus;

$\mathrm{Sb}$ : the branch innervating sternocleidomastoideus;

Sm: scalenus medius;

Ss: suprascapular nerve;

Tc: transverses colli;

Tr: trapezius;

$\mathrm{V}$ : ventral. 